\title{
" The Brave Sparks of the City » : Les apprentis dans les comédies citadines, citoyens de second ordre ou citoyens en devenir?
}

\section{Christophe Hausermann}

\section{(2) OpenEdition \\ 12 Journals}

\section{Édition électronique}

URL : http://journals.openedition.org/shakespeare/1623

DOI : $10.4000 /$ shakespeare. 1623

ISSN : 2271-6424

Éditeur

Société Française Shakespeare

\section{Édition imprimée}

Date de publication : 1 mars 2011

Pagination : 137-157

ISBN : 2-9521475-7-4

\section{Référence électronique}

Christophe Hausermann, « «The Brave Sparks of the City » : Les apprentis dans les comédies citadines, citoyens de second ordre ou citoyens en devenir? ", Actes des congrès de la Société française Shakespeare [En ligne], 28 | 2011, mis en ligne le 15 février 2011, consulté le 02 mai 2019. URL : http:// journals.openedition.org/shakespeare/1623 ; DOI : 10.4000/shakespeare.1623 


\section{Shakespeare et la Cité}

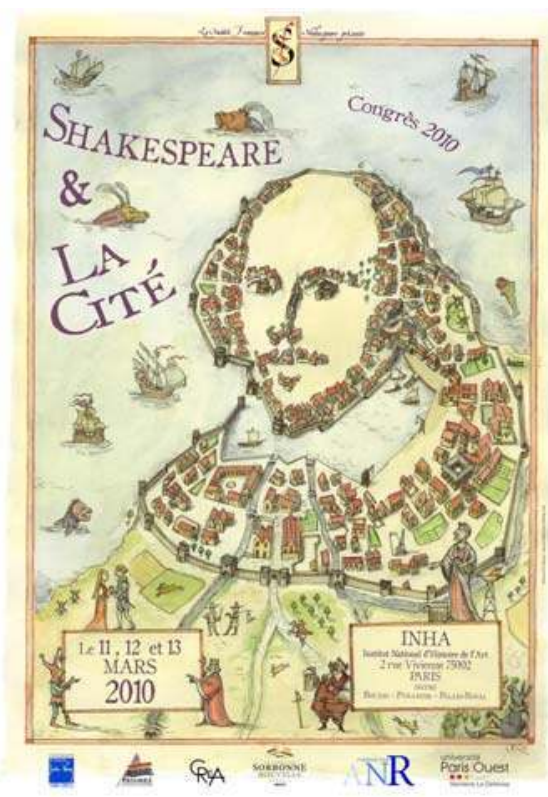

actes du Congrès

organisé par la

SOCIÉTÉ FRANÇAISE SHAKESPEARE

les 11,12 et 13 mars 10

textes réunis par

Pierre KAPITANIAK

sous la direction de

Dominique GoY-BLANQUET 
COUVERTURE :

Edouard Lekston 2010

conception graphique et logo

Pierre Kapitaniak

\section{(C) 2011 Société Française Shakespeare Institut du Monde Anglophone} Université de Paris III - Sorbonne Nouvelle 5 rue de l'École de Médecine 75006 Paris

www.societefrancaiseshakespeare.org réservés pour tous les pays 


\title{
« THE BRAVE SPARKS OF THE CITY 》 : LES APPRENTIS DANS LES COMÉDIES CITADINES, CITOYENS DE SECOND ORDRE OU CITOYENS EN DEVENIR ?
}

\author{
Christophe HAUSERMANN
}

\begin{abstract}
L'accession à la citoyenneté londonienne ne pouvait avoir lieu qu'à l'issue d'un long apprentissage. Après l'obtention de sa liberté et quelques années de compagnonnage, l'apprenti ambitionnait de devenir à son tour maître d'apprentissage et propriétaire de son atelier. Le système corporatiste assurait ainsi sa pérennité en régulant le comportement des apprentis. Dès lors, leur progression sociale dépendait de leur capacité à se plier au jugement du maître d'apprentissage et à patienter jusqu'à l'obtention de leur salutaire liberté. Les pièces de Thomas Dekker, Thomas Heywood, Philip Massinger et William Rowley ont transposé l'apprentissage sur scène et ont fait de l'apprenti un personnage de répertoire tour à tour veule et héroïque, fustigeant ses excès ou encensant ses exploits. Dans la représentation qu'elles donnent de l'apprentissage, les comédies citadines ont fidèlement décrit la vie de la Cité et de ses corporations. Elles ont également instauré une citoyenneté fantasmée accessible à tous ceux qui étaient prêts à fournir les efforts nécessaires pour l'obtenir.

'The Brave Sparks of the City': Apprentices in City Comedies - Would-be Citizens or Citizens to Be? Access to citizenship required a long apprenticeship. After the apprentice had been sworn a freeman and spent a few years as a journeyman, his ambition was to become in his turn a master and a householder. Thus, the system of livery companies perpetuated itself by controlling the apprentices' behaviour. So, the apprentices upward mobility depended on their ability to comply with their master's judgment and to be patient until they obtained their salutary freedom. Thomas Dekker's, Thomas Heywood's, Philip Massinger's and William Rowley's plays all stage the training of apprentices, thus making the apprentice a stock character, criticising his excesses and praising his high deeds. Through the representation of apprenticeship, city comedies have faithfully described the life of the City and that of its livery companies. They have also established a fantasized citizenship accessible to all those who were ready to make every endeavour to obtain it.
\end{abstract}

À l'époque élisabéthaine, un apprenti était un adolescent placé pour une durée moyenne de sept ans auprès d'un maître d'apprentissage. Il était souvent désigné par l'aphérèse "'prentice », comme dans le titre du manuel de bonne conduite The Pious Prentice, or the Prentice's Piety (1640) ${ }^{1}$. Ce mot était également orthographié «'prentise » comme dans le titre du recueil de poésie du futur Jacques I ${ }^{\text {er }}$ lorsqu'il n'était qu'apprenti poète, The Essayes of a Prentise in the Divine Art of Poesie (1585)². En France à la même époque, on déclinait encore le nom «apprenti» sous la forme «apprentif » au masculin et « apprentive » au féminin, et cela jusqu'au

${ }^{1}$ Abraham Jackson, The Pious Prentice, or, The Prentice's Piety, 1640, Londres, E. G[riffin], EEBO, Bodleian Library, STC (2nd ed.) / 14295.

2 Jacques I ${ }^{\mathrm{er}}$, The Essayes of a Prentise in the Divine Art of Poesie, Édimbourg, Thomas Vautroullier, 1584, EEBO, Henry E. Huntington Library and Art Gallery, STC (2nd ed.) / 14373 . 
XVII ${ }^{\mathrm{e}}$ siècle, comme l'atteste cette citation extraite des Caractères de La Bruyère qui dessine en creux la sujétion de l'apprenti et le mimétisme professionnel qui était attendu de lui : «Un apprentif est docile; il écoute son maître, il profite de ses leçons, et il devient maître ${ }^{3}$. »

Un apprenti commençait son apprentissage vers 19 ans et en sortait en moyenne à l'âge de 28 ans. Cependant, dans les faits un apprentissage pouvait parfois durer plus de dix ans. L'insertion dans la vie professionnelle ainsi que le mariage avaient lieu sur le tard, aux alentours de la trentaine. L'apprenti signait avec son maître un contrat d'apprentissage (indenture), aussi appelé contrat synallagmatique parce qu'il comportait une obligation réciproque entre les deux partis et imposait à chacun des devoirs, qui consistaient pour le maître à transmettre son savoir-faire et pour l'apprenti à obéir et à se comporter de façon exemplaire. L'apprenti devait prêter serment dans sa corporation et son contrat stipulait clairement que la fornication comme le mariage lui étaient formellement interdits durant toute la durée de son apprentissage. Chaque nouvel apprenti devait rédiger une petite profession de foi d'une cinquantaine de mots qui était ensuite consignée dans un recueil appelé Apprentices' Book of Oaths. Le nom de l'apprenti était alors inscrit dans les registres de la corporation d'origine de son maître.

À l'issue de son apprentissage, l'apprenti devenait un homme libre (freeman), c'est-à-dire un citoyen londonien. Cette citoyenneté était avant tout prospective, elle était à la fois une fin et une formidable source de motivation. Elle offrait à l'apprenti un fort sentiment d'appartenance communautaire et pourvoyait aux besoins de main d'œuvre de la Cité. Mais il arrivait que cette citoyenneté ne réponde pas aux espérances de l'apprenti, qu'elle soit contrariée par la dure réalité de la vie professionnelle. Il convenait donc à Londres et à ses corporations d'entretenir par tous les moyens possibles une citoyenneté fantasmée axée sur des promesses de prospérité, voire de postérité.

\footnotetext{
3 La Bruyère, « De la Chaire », 2, IV, Les Caractères, éd. Emmanuel Bury, Paris, Librairie Générale Française, 2004. La maxime se termine ainsi : "L’homme indocile critique le discours du prédicateur, comme le livre du philosophe, et il ne devient ni chrétien ni raisonnable. »
} 


\section{Une citoyenneté prospective}

Les apprentis étaient des citoyens en devenir qui se projetaient dans l'avenir en imaginant leur vie une fois obtenue leur liberté. Dans le chapitre intitulé «De l'institution des enfants » au premier livre des Essais, Montaigne déclare ceci : «Je ne vise icy qu'à découvrir moymesmes, qui seray par adventure autre demain, si nouvel apprentissage me change ${ }^{4}$. » L'apprentissage n'était pas tant un état qu'une évolution, le passage de la condition de novice à celle d'artisan confirmé, mais également à celle d'homme libre et de citoyen. Rien moins ne se joue dans l'apprentissage que le passage de l'adolescence à l'âge adulte et l'acquisition d'une identité sociale et citoyenne.

Un maître formait un apprenti qui, plus tard, deviendrait maître à son tour et perpétuerait le savoir-faire acquis lors de son apprentissage. Seul l'apprenti méritant pouvait espérer obtenir sa liberté après de longs efforts prodigués au service de son maître. L'apprenti n'était pas censé se poser en s'opposant, bien au contraire, il devait montrer son aptitude à reproduire les mêmes gestes que ceux de son maître en faisant preuve d'humilité. Comme le soulignait en 1927 le philosophe Alain dans Les Idées et les âges, il n'y a pas de place pour l'improvisation dans l'apprentissage, seule l'obéissance prévaut: «L'apprenti n'essaie pas, car la sévère loi des travaux le tient, et la nécessité n'a point d'égards. Une pièce préparée ou dégrossie se trouve gâtée par la moindre improvisation, surtout ingénieuse ; l'ordre des travaux réels est troublé ; le temps et la matière sont perdus ${ }^{5}$. » Ce qui était demandé à l'apprenti, c'était le respect de la consigne donnée par le maître ainsi que le respect des normes sociales édictées par les ordonnances de sa corporation et plus largement par les lois de la Cité. Il n'y avait nulle place pour l'individualité dans l'apprentissage, on y apprenait à se fondre dans la masse, à devenir un rouage dans la mécanique de la Cité, à acquérir une identité collective, celle de tous les apprentis de la corporation dans laquelle on exerçait.

Lors d'un séminaire à l'École Pratique des Hautes Études, Roland Barthes opposa l'apprentissage à deux autres pratiques, l'enseignement, dont la transmission s'effectue par le truchement d'un

${ }^{4}$ Michel de Montaigne, Les Essais, éds. Jean Balsamo, Michel Magnien et Catherine Magnien-Simonin, Paris, Gallimard, Bibliothèque de la Pléiade, 2007.

5 Alain (Émile Chartier), « Livre IV : Les Jeux », « Chapitre III : Le travail enfantin », Les Idées et les âges, Paris, Gallimard, 1927. 
discours, et le maternage, qui repose sur le soutien et l'encouragement. Selon Barthes, l'apprentissage se résume à la reproduction du geste :

le maître, donc, travaille pour lui-même devant l'apprenti ; il ne parle pas, ou du moins il ne tient pas de discours; son propos est purement déictique : "Ici je fais ceci pour éviter cela... » Une compétence se transmet silencieusement, un spectacle se monte (celui d'un faire), dans lequel l'apprenti, passant la rampe, s'introduit peu à peu 6 .

L'apprentissage relève en effet avant tout d'une monstration. L'enseignement du maître ne vaut que par la valeur de l'exemple. Mais le propos déictique avait dans le cadre de l'apprentissage londonien une double visée, il était non seulement maîtrise d'une pratique, ou pour reprendre l'expression de Barthes : «Ici je fais ceci pour éviter cela... », mais également éloge de la citoyenneté : « Ici je me comporte en tant que citoyen afin que tu le deviennes à ton tour ».

Pour autant, le maître d'apprentissage n'était pas un pédagogue, il n'avait pas vocation à faire de son apprenti un érudit, son enseignement était purement manuel, comme le souligne le compagnon cordonnier Barnabé à l'attention des nouveaux apprentis Crépin et Crépinien dans A Shoemaker, A Gentleman de William Rowley : « Bear up your head, boys, we'll teach ye to bristle, wax better and better, last to the 12 , then set foot in the stirrup ${ }^{7}$ and have at all ${ }^{8}$. » " Last to the twelve » signifie " effectuer une tâche jusqu'au bout ». Il s'agit aussi d'un jeu de mot, puisque " a last » est la forme de bois utilisée par les cordonniers et « 12 » représente la plus grosse pointure. Comme l'explique Barnabé en utilisant le vocable de son corps de métier, l'apprentissage est un perfectionnement perpétuel (« better and better ») jusqu'à l'obtention de la pureté du geste.

La citoyenneté n'était acquise qu'à la fin de l'apprentissage mais c'est elle qui lui donnait tout son sens car elle signifiait l'avènement de l'apprenti comme membre à part entière de la société londonienne. Un apprentissage réussi devait aboutir à l'autonomie de l'apprenti comme le rappelle le maître cordonnier dans A Shoemaker, A Gentleman :

\footnotetext{
${ }^{6}$ Roland Barthes, « Pratiques », texte sur le séminaire à l’École pratique des hautes études paru dans le numéro spécial de L'Arc consacré à l'auteur, reproduit dans Euvres complètes, vol. 4 (1972-1974), Paris, Seuil, 2002, p. 506.

${ }^{7}$ A stirrup : a shoemaker's strap, with which he keeps the last firm upon his knee (OED). En français, cette courroie est appelée « tire-pied ».

8 William Rowley, A Shoemaker, A Gentleman, éd. Trudi Darby, Londres, Nick Hern Books, 2002, I.ii.97.
} 
Well boys, on this condition I will entertain you ; I need not doubt your truths and honesty, you have such fair and promising outsides. But I must have you bound for seven years, and then you are your own men, and a good trade to get your livings by 9 .

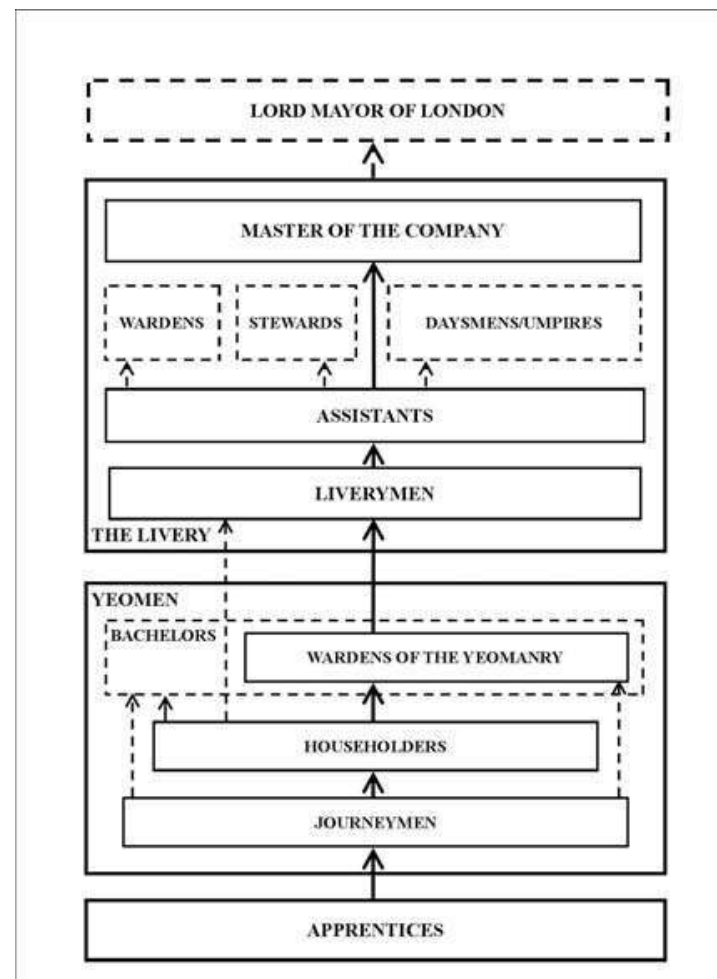

Figure 1 : Schéma hiérarchique d'une corporation londonienne

Les corporations promettaient à l'apprenti de gravir rapidement leurs différents échelons. L'apprentissage était le début d'un long parcours professionnel et chaque apprenti espérait un jour atteindre les plus hautes sphères de la vie corporatiste. S’il travaillait durement, un apprenti pouvait espérer accéder tour à tour aux cinq différents paliers corporatistes (the five estates): apprentices, journeymen,

${ }^{9}$ William Rowley, op. cit., I.ii.86-90 
householders, liverymen, assistants, que l'on retrouve sur le schéma hiérarchique ci-joint.

Après l'apprentissage avait lieu une période de compagnonnage de quelques années. Tout comme le terme "apprenti», celui de compagnon (journeyman) avait une connotation négative. C'est dans ce sens qu'Hamlet l'emploie lorsqu'il critique les acteurs médiocres : "I have thought some of Nature's journeymen had made men, and not made them well, they imitated humanity so abominably ${ }^{10}$. » Certaines corporations exigeaient un nombre minimum d'années de compagnonnage avant qu'un compagnon puisse travailler à son compte en ouvrant à son tour un atelier, devenant propriétaire (householder). Dans son ouvrage de référence Worlds within Worlds, Steve Rappaport cite l'exemple de la corporation des plâtriers (plasterers) qui en 1595 exigea de ses membres qu'ils effectuent au moins une année de compagnonnage. L'année suivante cette corporation rappela à l'ordre un compagnon nommé Robert Saunders en l'avertissant que s'il ne se pliait pas à cette exigence, «extremist punishment shall be inflicted upon him $^{11}$.»

Les compagnons et les propriétaires d'ateliers formaient «the yeomanry », appelée aussi «the freedom». Les yeomen ne faisaient pas partie des instances décisionnelles des corporations et y jouaient donc un rôle purement consultatif. Certains yeomen pouvaient exercer le poste de préposé aux affaires de la corporation (warden). Il fallait se montrer digne de confiance et avoir œuvré pour la communauté afin d'occuper ce poste.

Après une dizaine d'années de loyaux services au sein de sa corporation, un artisan pouvait accéder au poste tant convoité de liveryman qui, comme son nom l'indique, avait seul le privilège de porter la livrée honorifique de sa corporation, vêtement somptueux taillé dans les plus belles étoffes. Selon Steve Rappaport, seulement un cinquième des membres d'une corporation, et seulement un tiers des householders accédaient à la livrée ${ }^{12}$. Après une dizaine d'années supplémentaires, un liveryman pouvait devenir assistant, équivalent

${ }^{10}$ William Shakespeare, Hamlet, éd. Ann Thompson et Neil Taylor, The Arden Shakespeare, Third Series, Walton-on-Thames, Thomson Learning, 2005, III.ii.32-35.

${ }^{11}$ Steve Rappaport, Worlds within Worlds: Structures of Life in Sixteenth-Century London, Cambridge Studies in Population, Economy and Society in Past Time, Cambridge, C.U.P., (1989), 2002, p. 369 .

12 Rappaport, op. cit., p. 23 
d'un juré appartenant à la jurande des corporations françaises. Cette charge était conférée à un membre de la corporation pour la représenter et gérer les litiges internes au sein de la Court of Assistants. La Court of Assistants permettait de résoudre les conflits au sein même de la corporation, ce qui évitait d'avoir recours à une autre instance juridique comme la Court of Aldermen. Il était possible de faire appel de la décision de la Court of Assistants auprès de daysmen et autres umpires qui étaient désignés parmi les assistants. Les stewards quant à eux étaient des intendants dont les tâches consistaient entre autres à gérer des élections internes ou à organiser un banquet au sein de la corporation.

Avec l'appui de ses pairs, un membre riche et influent pouvait être élu maître de sa corporation et même accéder à la gloire en devenant Lord Maire. Tout apprenti était un citoyen en devenir à qui l'on promettait les plus hautes distinctions. Le schéma hiérarchique d'une corporation montre qu'il existait une voie royale pour gravir l'un après l'autre les échelons mais qu'il existait également de nombreux chemins de traverse.

\section{Une citoyenneté contrariée}

Les corporations offraient ainsi à leurs membres un parcours extrêmement balisé et leur faisaient miroiter une réussite professionnelle et sociale qui serait proportionnelle au zèle dont ils sauraient faire preuve. L'accession à la citoyenneté était avant tout pour l'apprenti une promesse de prospérité. Dans son édition des deux parties d'Edward IV de Thomas Heywood, Richard Rowland parle de « carrot of meteoric social elevation [...] dangled before recalcitrant apprentices ${ }^{13} »$. La naïveté des apprentis était un ressort classique des comédies citadines. Dans The City Madam de Philip Massinger, Luke Frugal a compris qu'il pourrait corrompre les apprentis Tradewell et Goldwire en leur promettant une situation professionnelle à la hauteur de leur mérite :

LUKE. Have you almost serv'd out

The term of your indentures, yet make conscience

By starts to use your liberty?

13 Thomas Heywood, The First and Second Parts of King Edward IV, éd. Richard Rowland, The Revels Plays, Manchester, Manchester University Press, 2005 


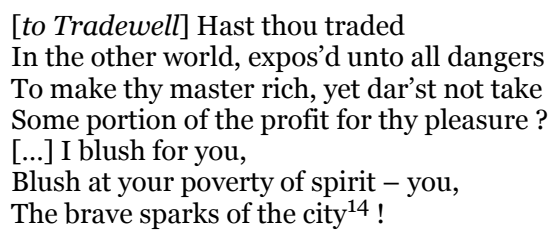

Si l'expression « The brave sparks of the city » utilisée par Luke Frugal peut paraître flatteuse de prime abord, elle sous-entend également que ces apprentis avaient des velléités de signer l'élégance vestimentaire de la noblesse, le mot «spark» signifiant selon l'Oxford English Dictionary : «A young man of an elegant or foppish character; one who affects smartness or display in dress and manners. Chiefly in more or less depreciatory use. » De même, l'adjectif « brave », s'il exprime le courage, désigne plutôt ici le fait d'être bien habillé. C'est dans ce sens que Thomas Heywood l'emploie dans An Apology for Actors : «A gentle person this, and he a clown / One man is ragged, and another brave. All men have parts, and each man acts his own ${ }^{15}$. " Sous couvert d'éloges, Luke déprécie les deux apprentis avec une certaine ironie. Au contraire, l'épicier de The Knight of the Burning Pestle, de Francis Beaumont, utilise ces mêmes termes dans un sens plus littéral pour designer son apprenti Rafe :

RAFE. I warrant you, mistress, if I do it not for the honour of the city and the credit of my master, let me never hope for freedom ;

WIFE. 'Tis well spoken i'faith. Go thy ways ; thou art a spark indeed.

Citizen. Rafe, Rafe, double your files bravely ${ }^{16}$.

Pour motiver un apprenti, il suffisait de flatter son ego et de lui faire croire qu'il possédait un ascendant sur d'autres personnes, ce qui bien sûr n'était qu'illusoire.

Bien que l'apprentissage fût un formidable système de promotion sociale, il existait de fortes différences de statut entre les corporations londoniennes. Il convient à cet égard de distinguer les

14 Philip Massinger, The City Madam, éd. Cathy Shrank, Londres, Nick Hern Books, 2005, II.ii.45-59.

15 Thomas Heywood, "the Author to his Book », An Apology for Actors, Londres, 1612, EEBO, Folger Shakespeare Library, STC (2nd ed.) / 13309.

${ }^{16}$ Francis Beaumont, The Knight of the Burning Pestle, éd. Michael Hattaway, Londres, Ernest Benn, New Mermaids, 1969, v.v.66-70. 
corporations de marchands, comme les merciers, qui tirent leur nom du latin « merx » qui signifie « marchandise », et d'autres corporations plus artisanales, reposant entièrement sur une activité manuelle comme les cordonniers. Un apprenti issu d'une petite corporation avait moins d'occasions de gravir l'échelle sociale qu'un apprenti appartenant aux douze grandes corporations londoniennes (The Twelve Great Livery Companies) ${ }^{17}$. Par ailleurs, le milieu social de l'apprenti influait grandement sur son avenir professionnel. La période de compagnonnage était plus courte, voire inexistante, pour les fils de famille qui bénéficiaient de fonds suffisants pour payer le bail de leur atelier, acheter des outils et des matières premières et loger des apprentis.

Le statut social d'un maître était lui aussi un gage de qualité au sein d'une corporation. Un maître appartenant à la livrée était très recherché puisqu'il connaissait les dignitaires de sa corporation et occupait parfois un poste à responsabilité (warden ou assistant) qui lui permettait d'asseoir son autorité. Un apprenti avait plus de chance d'obtenir rapidement sa citoyenneté et de devenir propriétaire s'il bénéficiait du patronage d'une personne riche ou influente au sein de sa corporation d'origine.

Ainsi, tout en se voulant égalitaire le système corporatiste n'en demeurait pas moins élitiste. Le début de carrière pouvait donner l'illusion d'une progression professionnelle accessible à tous mais la sélection s'accentuait au fur et à mesure des échelons gravis au sein d'une corporation et l'accession à la livrée demeurait un goulot d'étranglement qui en interdisait l'accès au plus grand nombre.

Par ailleurs, la vie d'un citoyen londonien n'était pas aussi palpitante que ce que les corporations laissaient croire à leurs apprentis. Certains commerces artisanaux faisaient banqueroute et les dettes pouvaient rapidement s'accumuler. Dans The City Wit de Richard Brome, l'orfèvre Crasy croule sous les dettes et se voit humilié par sa belle-mère qui oppose la chute professionnelle de son gendre à l'ascension de son mari, un bouvier devenu juge :

${ }^{17}$ Ces douze corporations sont par ordre de préséance: The Mercers (merciers), The Grocers (épiciers), The Drapers (drapiers), The Fishmongers (poissonniers), The Goldsmiths (orfèvres, bijoutiers), The Taylors (tailleurs), The Skinners (peaussiers), The Haberdashers (passementiers), The Salters (sauniers), The Ironmongers (ferronniers), The Vintners (négociants en vin) et The Clothworkers (travailleurs du tissu). 
PYANNET. Here's my worshipful husband, Mr. Sneakup, that from a Grazier is come to be a Justice of the Peace: And, what, as an honest man? He grew to be able to give nine hundred pound with my daughter; and, what, by honesty? Mr. Sneakup and I are come up to live I'the City, and here we have lied these three years ; and what? For honesty? Honesty! What should the City do with honesty when 'tis enough to undo a whole Corporation ${ }^{18}$ ?

La belle-mère de Crasy lui démontre que l'honnêteté n'est pas un gage de réussite sociale, il vaut mieux être un arriviste sans scrupules comme son mari et s'enrichir en gravissant les différents niveaux de la hiérarchie plutôt que de s'endetter en restant fidèle à des convictions morales rétrogrades.

Les revers de fortune qu'un artisan pouvait rencontrer au cours de sa carrière n'entamaient pas sa confiance en l'avenir s'il persistait à croire à un dessein professionnel nourri par les traditions populaires. L'adage selon lequel «A shoemaker's son is a prince born » était connu de tous. On retrouve cette expression dans de nombreuses comédies citadines mais c'est dans The Shoemaker's Holiday de Thomas Dekker que l'on trouve le plus grand nombre d'occurrences puisque le cordonnier Simon Eyre l'utilise à six reprises ${ }^{19}$. Celui-ci s'enorgueillit d'être le fils unique d'un cordonnier, ce qui lui confère d'indubitables lettres de noblesse, et il ne se lasse pas de le dire à qui veut bien l'entendre: «Peace, am I not Simon Eyre? Are not these my brave men, brave shoemakers, all gentlemen of the Gentle Craft ? Prince I am none, yet am I nobly born, as being the sole son of a shoemaker ${ }^{20}$.

Simon Eyre considère également les cordonniers qui officient dans son atelier comme des gentilshommes puisque la cordonnerie est un noble métier, « a gentle craft ». Ce tautologie permettait de valoriser une profession et des ouvriers que rien ne prédisposait à une brillante carrière ou une grande renommée. Une fois encore, Simon Eyre utilise l'adjectif «brave » pour qualifier ses apprentis et compagnons afin de les mettre en valeur et de les galvaniser, espérant ainsi qu'ils redoublent d'efforts au travail. Cette bravoure qui revient comme un

\footnotetext{
18 Richard Brome, The City Wit, in Dramatic Works of Richard Brome Containing Fifteen Comedies, 3 vol., Londres, J. Pearson, 1873, rééd., New York, 1966, p. 284.

${ }^{19}$ En plus de l'extrait cité, le proverbe apparaît dans les passages suivants : x.136, xi.16, xvii.19, xxi.15 et 33 .

${ }^{20}$ Thomas Dekker et autres, The Roaring Girl and Other City Comedies, [The Shoemakers' Holiday, Eastward Ho !, Everyman in his Humour, The Roaring Girl], éd. James Knowles, Oxford, O.U.P., 2001, vii.40-43.
} 
leitmotiv à la bouche des maîtres d'apprentissage doit s'entendre comme une injonction à affronter le dur labeur et à en sortir victorieux. Bien que tout le monde s'accordât sur la nécessité d'un travail artisanal dans la Cité, Érasme rappelait dans ses Adages que « ne sutor ultra crepidam », ce que Richard Taverner traduisit en 1539 par : " Let not the shoemaker go beyond hys shoe ${ }^{21} »$. Il importait donc que chacun connaisse sa place dans la Cité et qu'il se borne à l'occuper durant toute son existence, même si cela impliquait d'être un citoyen de second ordre.

\section{Une citoyenneté fantasmée}

Pour motiver les apprentis et leur faire oublier la pénibilité du travail qui les attendait, certains récits populaires les assimilaient à d'héroïques combattants, cumulant les exploits chevaleresques. Les apprentis s'enrôlaient ainsi pour sauver leur Cité et leur pays et défendre l'honneur de leur monarque. Le titre du récit suivant est particulièrement élogieux: The Honour of London Apprentices : Exemplified, in a Briefe Historicall Narration; Containing Many Heroicall Actions Done by (Some in Particular, the Rest in Generall) Prentices of the Most Renowned, and (in that Regard) Truly Honourable City of London, Both at Home and Abroad. In Long, Fore-Past, Present, and Moderne Times. Collected and Published, by a Well-Wisher to What Himselfe Once Was that is to Say, a Prentice (and Now a Free Citizen) of the Famous City of London (1647) 22.

Il fallait également que les apprentis s'identifient à des citoyens légendaires qui avaient œuvré toute leur vie pour la prospérité de leur

\footnotetext{
${ }^{21}$ Érasme, Prouerbes or Adagies with Newe Addicions Gathered out of the Chiliades of Erasmus by Richard Taverner, London, 1539, EEBO, STC ( $2^{\text {nd }}$ ed.) $/ 10437$. Dans ce même recueil on trouve deux autres adages similaires : "Oportet remum ducere qui didicit. He oughte to holde $\mathrm{y}^{\mathrm{e}}$ oore $\mathrm{y}^{\mathrm{t}}$ hathe lerned it. That is to saye : Euery man muste practyse that science \& facultie, $\mathrm{y}^{\mathrm{t}}$ hath ben afore taughte him. Let not the shomaker medle further then hys shoes. Lette the ploughman talke of his plough. » et : "Quam quis\{que\} norit artem, in hac se exerceat. Let euerye man exercise hym selfe in the facultie that he knoweth. Let the kobler medle wyth clowtyng his neyghbours shoes, and not be a captaine in felde, or meddel wyth maters concernynge a common wealth. Let them iudge of controuersies in the christen religion, yt be lerned in the same, and not euery Iacke plowman. »

22 Voir également : The Honour of a London Prentice. Being an Account of his Matchless Manhood and Brave Adventures Done in Turkey, and by What Means he Married the King's Daughter, Londres, 1695, EEBO, British Library, C.20.f.9[747] et Londons Glory or, The Histor [y] of the Famous and Valiant London-Prentice, 1700, EEBO, Wing (CD-ROM, 1996) / S3508A.
} 
Cité alors qu'ils avaient commencé comme simples apprentis. Tous les apprentis londoniens connaissaient l'histoire de l'apprenti mercier Richard Whittington, dont le nom est mentionné dans de nombreuses comédies citadines ${ }^{23}$ et dont Thomas Heywood a retracé la fulgurante carrière dans une hagiographie qui mêle faits avérés et légendes urbaines intitulée The Famous and Remarkable History of Sir Richard Whittington, Three Times Lord-Mayor of London. Dans la même veine élogieuse, une ballade intitulée London's Glory, and Whittington's Renown or, A Looking-Glass for Citizens of London insistait particulièrement sur l'émulation qu'inspirait un si grand homme :

Brave London Prentices,

Come listen to my Song

'Tis for your glory all,

And to you doth belong,

And you poor Country Lads,

Though born of low degree,

See by god's providence,

What you in time may be ${ }^{24}$.

Richard Whittington devint dès le $\mathrm{XVI}^{\mathrm{e}}$ siècle un personnage populaire appelé « Dick» Whittington. Selon la version consacrée, Dick Whittington était un apprenti qui acquit une fortune grâce à son chat. Il donna celui-ci en gage sur un bateau partant pour le grand large et devint riche quand le chat fut acheté par le roi de Barbarie dont le palais était infesté par les rats. Il épousa alors la fille de son maître. Puis, devenu Lord Maire, il défila dans les rues de Londres. Enfin, il finança de nombreux chantiers urbains tels que l'extension de Guildhall ou l'hospice Whittington College. Il finança également la reconstruction de Guildhall, de l'église de St Michael Paternoster Royal, de la prison de Newgate, de la bibliothèque des Grey Friars, d'une partie de l'hôpital de St. Bartholomew et il créa un refuge pour mères célibataires au St Thomas Hospital, des systèmes de canalisation

\footnotetext{
23 Dick Whittington est cité comme exemple par le bijoutier Touchstone dans Eastward Ho! de Chapman, Marston et Jonson: «I must tell thee, I hope to see thee one o'the monuments of our City, and reckoned among her worthies, [...] when the famous fable of Whittington and his puss shall be forgotten ». Dick Whittington est également cité dans The Knight of the Burning Pestle de Francis Beaumont et dans 2 If You Know not Me, You Know Nobody de Thomas Heywood, deux autres pièces mettant en scène des apprentis.

${ }^{24}$ London's Glory, and Whittington's Renown or, A Looking-Glass for Citizens of London, Londres, 1641, EEBO, Wing (2nd ed.) / L2930.
} 
dans les quartiers de Billingsgate et Cripplegate, et des toilettes publiques dans la paroisse de St Martin Vintry. Par l'intermédiaire de ses généreux legs urbains, le citoyen prospère marquait durablement la Cité de sa présence et se rappelait au bon souvenir de ses concitoyens, assurant par la même sa célébrité posthume.

William Walworth est un autre de ces apprentis promus à une brillante carrière. Apprenti poissonnier, il devint Lord Maire de Londres en 1381. Il défendit le roi Richard II contre la jacquerie de Jack Straw. Walworth est mentionné par John Stow dans ses Chroniques ${ }^{25}$ et figure également dans The Nine Worthies of London ${ }^{26}$ de Richard Johnson ainsi que dans la pièce anonyme The Life and Death of Jack $S t r a w^{27}$. Selon la légende, authentifiée par Holinshed mais contredite par Stow, la dague rouge qui figure sur les armoiries de Londres commémorerait l'exécution du rebelle Wat Tyler par William Walworth. Thomas Heywood cite cette action comme exemple de bravoure dans la première partie d'Edward $I V^{28}$ ainsi que dans la seconde de If You Know not Me, You Know Nobody ${ }^{29}$. Anthony Munday fit, quant à lui, ressusciter Walworth dans le spectacle écrit pour célébrer le nouveau Lord Maire en 1616, Chrysanaleia: the Golden Fishing ${ }^{30}$. En effet, au cours de la procession du Lord Maire, Walworth sortit de sa tombe et vint narrer ses actes de bravoure, immortalisés par les armoiries de la ville :

And as my Dagger slew the Rebell then,

So to renowne the deede ; And I dare say,

To honor London more (if more it may)

The Red-Crosse, in a Silver-field before,

Had Walworth's Dagger added to it more.

\footnotetext{
25 John Stow, The Chronicles of England from Brute vnto this Present Yeare of Christ. 1580, Londres, Henry Bynneman, 1580, EEBO, STC (2nd ed.) / 23333.

${ }^{26}$ Richard Johnson, The Nine Worthies of London, 1592, EEBO, STC (2nd ed.) / 14686

27 The Life and Death of Iacke Straw, a Notable Rebell in England Who was Kild in Smithfield by the Lord Maior of London, Londres, 1594, EEBO, STC (2nd ed.) / 23356.

28 Thomas Heywood, op. cit., scène 5, 6-8.

29 Thomas Heywood, The Second Part of If You Know not Me, You Know no Bodie with the Building of the Royall Exchange: and the Famous Victorie of Queene Elizabeth, in the Yeare 1588, Londres, Thomas Purfoot, 1606, EEBO, STC (2nd ed.) / 13336.

$3^{0}$ Voir l'analyse que fait Tracey Hill de ce pageant dans Anthony Munday and Civic Culture : Theatre, History and Power in Early Modern London 1580-1633, Manchester, Manchester University Press, 2004, édition de poche, 2009.
} 
Walworth convoquait ensuite dans son discours en l'honneur du Lord Maire toutes les vertus royales qui étaient supposées être les siennes, vertus incarnées par des figurants sur le Char de la Victoire qui enjoignaient les citoyens de Londres à se comporter de façon exemplaire :

Truth, Vertue, Honour, sober Temperance, Fortitude, Zeale, Equitie, Conscience, Iustice, Authoritie, carefull Uigilance, Peace, Plenty, Law, Councell, Obedience, And Discipline, that whips all Errors hence, These (as best Pillars) do support this State: And euery Kingdome else doth propagate ${ }^{31}$.

Dans la deuxième partie d'If You Know not Me, You Know Nobody de Thomas Heywood, le docteur Nowell invite chez lui le mercier Thomas Gresham et le passementier Hobson afin de leur montrer sa galerie de portraits de citoyens londoniens célèbres. Parmi eux figurent notamment les portraits de Richard Whittington et de William Walworth. Le docteur Nowell n'a de cesse de vanter la bravoure de ce dernier :

DR. NOWELL. That prouident, valiant, and learned Citizen, That both attacht, and kild the traytor Tyler,

For which good seruice Walworth the Lord Maior,

This Felpot, and foure other Aldermen,

Were knighted in the field.

Thus did he liue, and yet before he di'd

Assur'd reliefe for thirteene poore for euer.

GRESHAM. By the marry God a worthy Citizen ${ }^{32}$.

Les corporations accrochaient elles aussi dans leur salle commune les portraits de leurs membres les plus prestigieux. Tout dans la représentation de l'homme de corporation devait souligner la réussite professionnelle afin que, par mimétisme, les apprentis reproduisent les hauts faits d'un citoyen d'exception. Baldassare Castiglione dans Le Livre du courtisan (1528) déclare que: "Quiconque veut donc être un bon disciple doit non seulement faire bien les choses, mais aussi toujours mettre toute son application à

\footnotetext{
${ }^{31}$ Munday, Anthony, Chrysanaleia: the Golden Fishing: or Honour of Fishmongers, 1616, EEBO, STC (2nd ed.) / 18266.

$3^{2}$ Thomas Heywood, The Second Part of If You Know not Me, You Know no Bodie, [I.i.]771-779.
} 
ressembler à son maître, et, si possible, à se transformer en lui ${ }^{33}$. » Cependant, la posture du marchand désireux de léguer son portrait à sa corporation est bien loin de la désinvolture (sprezzatura) qui est selon Castiglione l'une des vertus essentielles de l'homme de cour. Au contraire, la représentation picturale du marchand est le signe d'un désir ostentatoire, d'une vaine gloire, dénoncée par Francis Bacon dans ses Essays or Counsels, Civil and Moral (1625) ${ }^{34}$. Le but de ce genre de portrait était de montrer que le succès n'était pas fortuit et qu'il était le résultat d'un travail constant. Les marchands les plus riches et

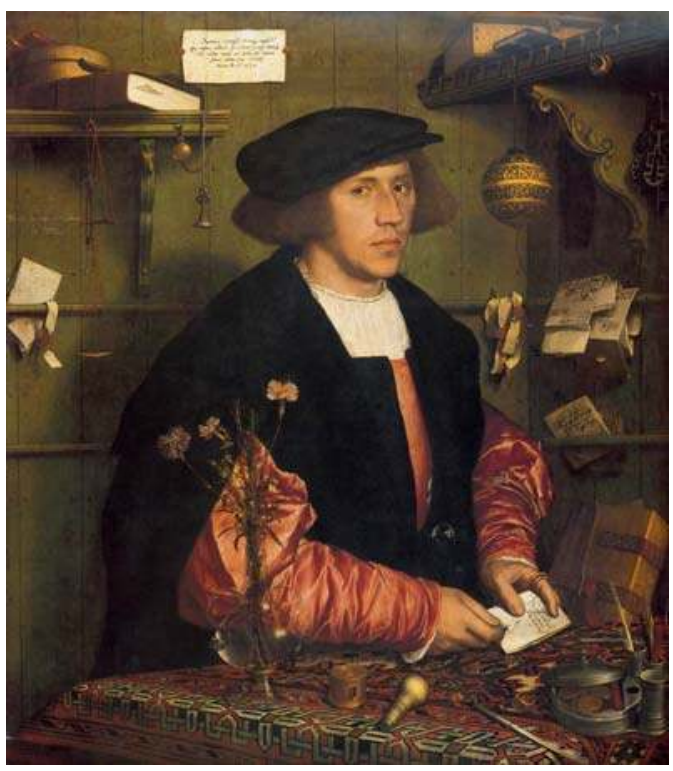

Figure 2 : Portrait du marchand Georg Gisze par Hans Holbein (1532), Gemäldegalerie, Berlin

influents faisaient don de leur portrait à leur corporation afin que leur réussite professionnelle inspire tous leurs membres, en particulier les apprentis.

\footnotetext{
33 Baldassar Castiglione, Le Livre du courtisan, éd. et trad. Alain Pons, Paris, Flammarion, 1991, p. 53.

34 Francis Bacon, « Of Vain-Glory », The Major Works, éd. Brian Vickers, Oxford, O.U.P., 1996, nouvelle éd., 2002, p. 443.
} 
Le portrait du marchand hanséatique Georg Gizse peint par Hans Holbein le jeune en 1532 est particulièrement révélateur des aspirations à la grandeur de la bourgeoisie du XVI siècle. Dans le coin inférieur droit du tableau figurent des pièces de monnaie, symbole de réussite financière, une écritoire, divers sceaux ainsi qu'une paire de ciseaux qui pouvait notamment servir à découper un contrat
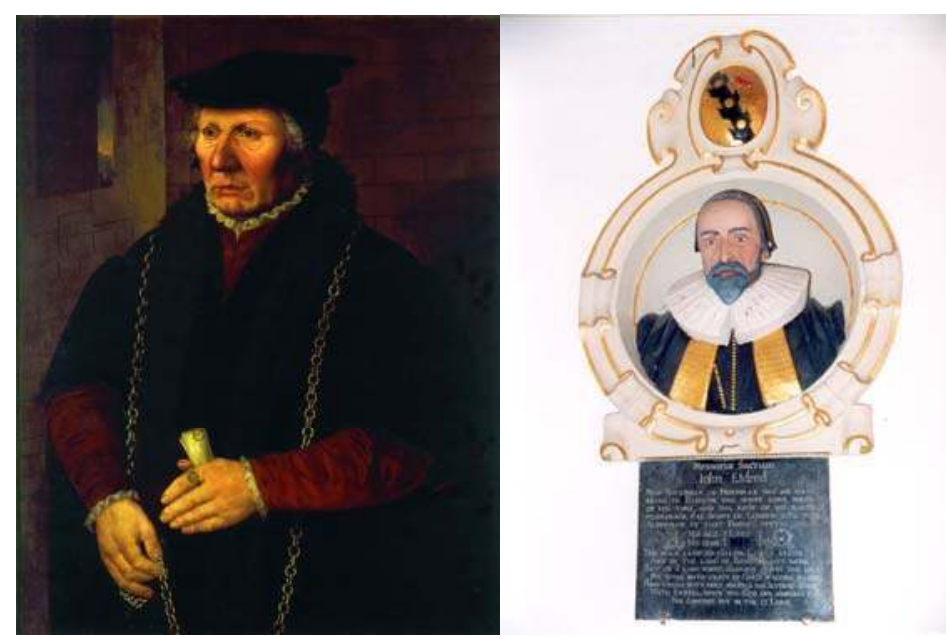

Figure 3 : Portrait du travailleur du tissu William Hewitt, Lord Maire de Londres en 1559, reproduit avec l'aimable autorisation de la Clothworkers'Company

Figure 4 : Buste de John Elred, Maître de la corporation des travailleurs du tissu en 1604, reproduit avec l'aimable autorisation de la Clothw orkers'Company

d'apprentissage. Georg Gizse tient dans ses mains une enveloppe qui est adressée à son nom : « À transmettre à mon frère, l'honorable Georg Gisze à Londres en Angleterre ». L'honneur du marchand est ainsi signifié sur le portrait, mais il est rapporté par une tierce personne, ce qui le légitime et balaie tout soupçon de vanité.

Tout aussi ostentatoires, le portrait du travailleur du tissu William Hewitt, membre de la corporation des travailleurs du tissu (Clothworkers) et Lord Maire de Londres en 1559 et le buste de John Elred, maître de cette même corporation en 1604, semblaient rappeler aux apprentis qu'il était possible d'acquérir une grande renommée s'ils 
s'en donnaient la peine. À l'inverse des portraits aristocratiques, les portraits de riches marchands visaient non pas à exhiber de somptueuses tenues ou divers attributs nobiliaires, mais plutôt à prôner une citoyenneté épanouie, au travers de laquelle ces marchands pouvaient acquérir une reconnaissance publique et une place honorifique dans la Cité.

Dans son article «The Arts and Acts of Memorialization in Early Modern London », Ian Archer mentionne le cas d'un tonnelier qui fit don de son portrait à sa corporation :

George Swayne, cooper (1607), left to his company "my picture now remayninge at the painters", with the condition that the picture be set up continually together with a table showing all the money, linen, and books he had given to the company, "to this only intent and consideracion that other members of the said company may bee therby incouraged to doe the like and not of any vaine glorious showe or ostentacion but to shewe my good will and affection to that societye 35 ".

Bien qu'il se défendît d'être vaniteux, ce tonnelier désirait assurer sa postérité au sein de sa corporation en dissimulant sa vainegloire derrière une posture désintéressée. Pourtant, la charité des marchands s'inscrivait avant tout dans une idéologie de la représentation. Ces peintures représentant les membres éminents d'une corporation participaient de ce que Stephen Greenblatt nomme «selffashioning ${ }^{6}$ ». Ce besoin d'ostentation sublimait l'activité manuelle de l'artisan car celle-ci dépréciait généralement son engagement citoyen.

Les corporations avaient également en leur possession de magnifiques pièces d'argenterie, les livery plates, qui étaient des assiettes et des coupes en argent ou en or fabriquées grâce au legs d'un généreux liveryman. Ces précieux objets portaient souvent le blason ou le nom de leur donateur, ce qui montre une fois encore que cette généreuse démarche n'était pas totalement désintéressée. Ces livery plates étaient exposées dans le grand hall des corporations afin que tous leurs membres puissent admirer leur raffinement et la munificence de leur donateur. Ses signes de richesse étaient aussi censés encourager les apprentis à émuler les membres les plus

35 Cité dans Ian W. Archer, «The Arts and Acts of Memorialization in Early Modern London », in J. F. Merritt, éd., Imagining Early Modern London, Cambridge, C.U.P., 2001, p. 97.

${ }^{36}$ Stephen Greenblatt, Renaissance Self-Fashioning, From More to Shakespeare, Chicago, University of Chicago Press, 1980. 
prospères de leur corporation. Il faut imaginer l'attrait de telles pièces pour de simples apprentis dont le quotidien consistait pour la plupart à travailler dans un atelier modeste. L'exhibition ostentatoire de ces pièces d'argenterie lors de grandes occasions devait probablement stimuler leur envie de devenir eux aussi d'éminents citoyens adulés de tous.

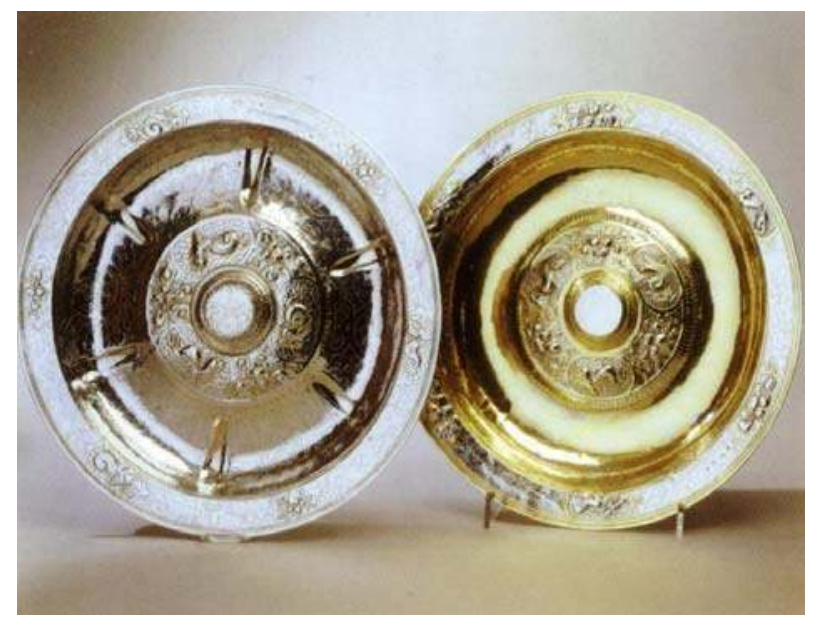

Figure 5 : The Clothw orkers'plates, reproduites avec l'aimable autorisation de la Clothw orkers' Com pany

Bien que ces pièces d'argenterie fussent surtout utilisées à des fins de décoration, elles pouvaient également servir dans les grandes occasions, lors de l'élection d'un nouveau maître au sein de la corporation ou lors de fêtes religieuses, comme la Fête-Dieu (Corpus Christi). De nombreuses livery plates ont été vendues ou fondues par les corporations pour rembourser leurs dettes mais certaines pièces d'époque sont encore utilisées aujourd'hui. C'est le cas notamment des Cockayne Cups de la corporation des peaussiers (skinners) qui furent créées grâce au legs de 120 livres que le marchand William Cockayne coucha dans son testament. Ces coupes sont encore utilisées lors de l'élection du nouveau maitre de la corporation suivant un rituel codifié dans ses moindres détails :

The heads are removable and the bodies are filled with wine and used to drink the health of the new master and wardens in the ceremony of the 
Cocks and Caps. In this ceremony newly elected members of the livery parade the Cockayne cups and five caps, velvet circlets adorned with Company emblems, around the central table in the banqueting hall. The new livery members are usually led by regimental bandsmen so it is a noisy and colourful affair. The procession stops and the outgoing master is given a cap which he places askew on the head of the chief guest ; everybody boos loudly as it does not 'fit'. When it is placed snugly on the new master's head everybody cheers. This part of the ceremony is thought to be one of the last surviving examples of a common ritual and the origins of the phrase 'if the cap fits wear it'. The master and master elect then drink each other's health from a cup and each new warden is crowned with a cap and is toasted from a Cockayne cup in a similar fashion 37 .

Cette cérémonie évoque bien entendu un faux couronnement qui permettait de conférer un apparat nobiliaire à un modeste rite corporatiste.

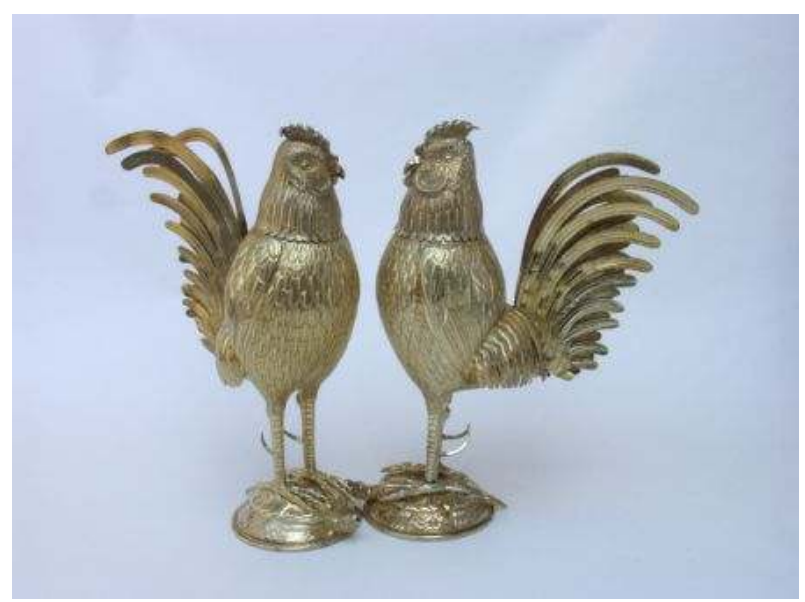

Figure 6 : The Skinners'plates: The Cockaigne Cups, reproduites avec l'aimable autorisation de la Skinners' Com pany

Il ne tenait qu'aux apprentis de devenir aussi riches et adulés que les membres les plus distingués de leur corporation et d'accéder à la reconnaissance de la Cité et de tous ses habitants. Les Whittington Ordinances de 1442, appartenant à la corporation des merciers, présentent une illustration sur laquelle Richard Whittington est allongé

${ }^{37}$ Site internet de The Skinners' Company : http://www.skinnershall.co.uk/history/thecockayne-cups.htm 


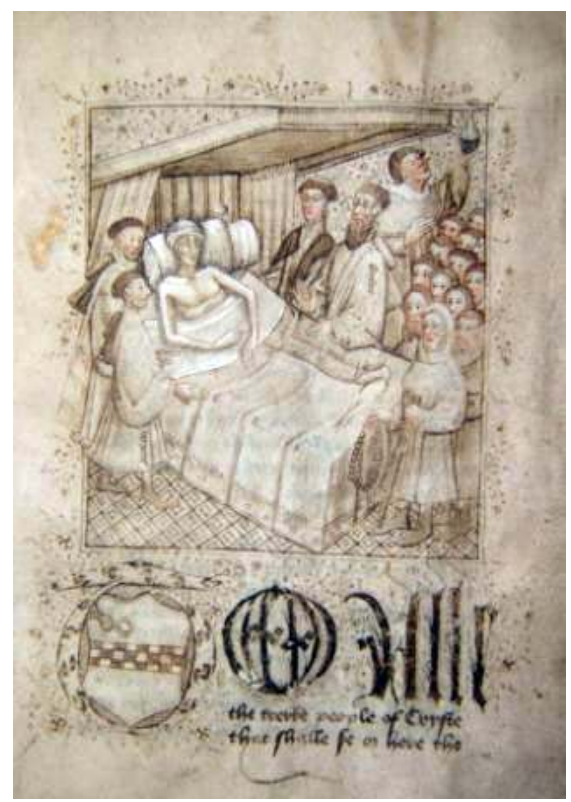

Figure 7 : Richard Whittington on his Deathbed, mars 1423 ,

illustration extraite des Whittington Ordinances de 1442,

reproduite avec l'aimable autorisation de la Mercers' Co m p a ny

sur son lit de mort ${ }^{3}$. Après une vie passée au service de la communauté, Whittington pouvait enfin trouver son salut. Le marchand revêt ici des allures de saint vénéré par tous les Londoniens et l'on pourrait presque imaginer que cette adoration de la population était une sorte de « canonisation citoyenne».

L'identité citoyenne d'un apprenti ne pouvait se constituer qu'à partir d'éléments traditionnels disparates d'où émergeait une identité fantasmée, entretenue par une littérature populaire et des rites corporatistes savamment orchestrés. Une citoyenneté sublimée se substituait ainsi à la citoyenneté coercitive qui exigeait des apprentis un travail acharné et une obéissance constante. Leur progression sociale dépendait de leur capacité à se plier aux règles de la vie

38 « The only portrait of Whittington at all likely to be authentic » (Dictionary of National Biography). 
communautaire et à patienter jusqu'à l'obtention de leur salutaire liberté.

L'apprenti devait se fondre dans la ville. En devenant citoyen, il faisait ainsi corps avec la Cité qu'il incarnait par substitution métonymique. Dans A Survey of London, John Stow assimile la Cité à une sorte de Leviathan urbain constitué de tous ses citoyens, comme s'il s'agissait d'un être animé par l'activité professionnelle de ses commerçants et artisans : "It is besides the purpose, to dispute, whether the estate of the gouernment here bee a Democratie, or Aristocratie, for whatsoeuer it bee being considered in it selfe, certaine it is, that in respect of the whole Realme, London is but a Citizen ${ }^{39}$. »

Christophe HAUSERMANN Université de Paris III Sorbonne Nouvelle

39 John Stow, « The Singularities of the City of London », in The Survey of London, 1598, $E E B O$, STC (2nd ed.) / 23341, p. 478. 